\title{
Glucose Plus Insulin Regulate Fat Oxidation by Controlling the Rate of Fatty Acid Entry into the Mitochondria
}

\author{
Labros S. Sidossis, ${ }^{\star \star \star}$ Charles A. Stuart, " Gerald I. Shulman, ${ }^{\ddagger}$ Gary D. Lopaschuk, ${ }^{\S}$ and Robert R. Wolfe ${ }^{\star \mid \star \star \star}$ \\ $*$ Metabolism Unit, Shriners Burns Institute, Galveston, Texas 77550; ${ }^{\ddagger}$ Department of Internal Medicine, Section of Endocrinology, Yale \\ University, New Haven, Connecticut 06520; ' Cardiovascular Disease Research Group and Lipid Lipoprotein Research Group, \\ Department of Pediatrics and Department of Pharmacology, University of Alberta, Edmonton T6G 2S2, Canada; and $\|_{\text {Department of }}$ \\ Anesthesiology, "Department of Internal Medicine, and **Department of Surgery, University of Texas Medical Branch, Galveston, \\ Texas 77550
}

\begin{abstract}
We tested the hypothesis that glucose plus insulin determine the rate of fat oxidation in humans by controlling the rate of fatty acid entrance into the mitochondria. We gave constant infusions of $\left[1-{ }^{13} \mathrm{C}\right]$ oleate, a long-chain fatty acid, and $\left[1-{ }^{14} \mathrm{C}\right]$ octanoate, a medium-chain fatty acid, for $3 \mathrm{~h}$ in seven volunteers (basal). Immediately after the basal period, a hyperinsulinemic (insulin infusion $=120 \mathrm{mU} \cdot \mathrm{m}^{-2}$. $\min ^{-1}$ ), hyperglycemic (plasma glucose $=140 \mathrm{mg} / \mathrm{dl}$ ) clamp was started and continued for $5 \mathrm{~h}$. During the last $3 \mathrm{~h}$ of the clamp, the infusions of $\left[1-{ }^{13} \mathrm{C}\right]$ oleate and $\left[1-{ }^{14} \mathrm{C}\right]$ octanoate were repeated. Intracellular acylcarnitine concentrations were measured in muscle biopsies obtained before and after the clamp. Plasma oleate enrichment and FFA concentration were kept constant by means of variable infusions of lipids and heparin. Oleate, but not octanoate, requires carnitine binding to gain access to the mitochondrial matrix; hence, if glucose and/or insulin limit long-chain fatty acid entrance into the mitochondria, then, during the clamp, long-chain acylcarnitine formation should be decreased, causing a decrease in oleate, but not octanoate, oxidation. Oleate oxidation decreased from the basal value of $0.7 \pm 0.1$ to $0.4 \pm 0.1 \mu \mathrm{mol} \cdot \mathrm{kg}^{-1} \cdot \mathrm{min}^{-1}(P<0.05)$. In contrast, octanoate oxidation remained unchanged. Long-chain acylcarnitine concentration decreased from $855 \pm 271$ in the basal state to $376 \pm 83 \mathrm{nmol} / \mathrm{gram}$ dry weight during the clamp $(P<0.05)$. We conclude that glucose and/or insulin determine fatty acid oxidation by controlling the rate of long-chain fatty acid entrance into the mitochondria. ( $J$. Clin. Invest. 1996. 98:2244-2250.) Key words: medium chain fatty acids - malonyl-coenzyme A - carnitine palmitoyltransferase $\cdot$ diabetes $\bullet$ obesity
\end{abstract}

\section{Introduction}

Since the introduction of the concept of fatty acid-mediated inhibition of glucose oxidation (1), several investigators have

Address correspondence to Dr. Labros S. Sidossis, Metabolism Unit, Shriners Burns Institute, University of Texas Medical Branch, Galveston, TX 77550. Phone: 409-770-6622; FAX: 409-770-6626; E-mail: labros@utmbsbisparc.utmb.edu

Received for publication 9 July 1996 and accepted in revised form 19 September 1996.

J. Clin. Invest.

(C) The American Society for Clinical Investigation, Inc. 0021-9738/96/11/2244/07 \$2.00

Volume 98, Number 10, November 1996, 2244-2250 examined the potential effect of elevated fatty acid availability and oxidation on glucose uptake and disposal in various tissues (e.g., 2-7). However, the effect of glucose availability and oxidation on fatty acid oxidation has not received similar attention. When glucose availability increases, fatty acid oxidation decreases (e.g., 6, 8). This response is mediated, at least in part, via an insulin-induced inhibition of lipolysis, resulting in decreased plasma FFA availability. However, as we have shown recently, hyperglycemia-hyperinsulinemia may directly inhibit fatty acid oxidation even when plasma fatty acid availability is maintained constant (9). The finding that high glycolytic flux inhibits fatty acid oxidation directly, a notion reverse of the socalled "glucose-fatty acid cycle" hypothesis (1), suggests that glucose and/or insulin exerts its effect intracellularly. However, the exact mechanism via which glucose and/or insulin regulate fatty acid oxidation in humans is still unclear.

A potential mechanism by which increased glucose metabolism could directly inhibit fatty acid oxidation has been proposed for the liver (10). Activated long-chain fatty acids $(\mathrm{LCFA})^{1}$ must bind to carnitine, a reaction catalyzed by the enzyme carnitine palmitoyl-transferase I (CPT I), to gain access into the mitochondrial matrix (11) (Fig. 1). Based on findings from studies in rat livers in vitro it has been suggested that in the carbohydrate fed state, when the insulin to glucagon ratio increases, the decrease in cytosolic cyclic $3^{\prime}, 5^{\prime}$-adenosine monophosphate concentration, coupled with an increase in pyruvate availability, results in increased formation of malonyl-CoA $(10,12,13)$, a potent inhibitor of CPT I (10). Thus, when cytosolic malonyl-CoA concentration increases, LCFA uptake by the mitochondria is inhibited (10), and as a consequence, fatty acid oxidation decreases. As starvation progresses (low insulin to glucagon ratio), malonyl-CoA concentration decreases which relieves the inhibition on CPT I, thereby allowing the flow of long-chain fatty acyl-CoA into the mitochondria for oxidation.

Direct evidence to support the notion that the controlling site for fatty acid oxidation is the CPT I reaction has come from experiments in isolated perfused rat livers. It was shown that unlike LCFA, octanoate, a medium-chain fatty acid (MCFA), was oxidized to acetyl-CoA at similar rates in livers from fed, fasted, and diabetic rats and the rate of octanoate conversion to ketones was similar in all three groups (14). Since, unlike LCFA, MCFA can diffuse freely through the in-

1. Abbreviations used in this paper: CPT I, carnitine palmitoyl-transferase I; LCFA, long-chain fatty acids; MCFA, medium-chain fatty acid; $R_{\mathrm{a}}$, rate of appearance; $R_{\mathrm{d}}$, rate of disappearance; TG, triacylglycerol; $\mathrm{VCO}_{2}$, carbon dioxide production; $\mathrm{VO}_{2}$, oxygen consumption. 


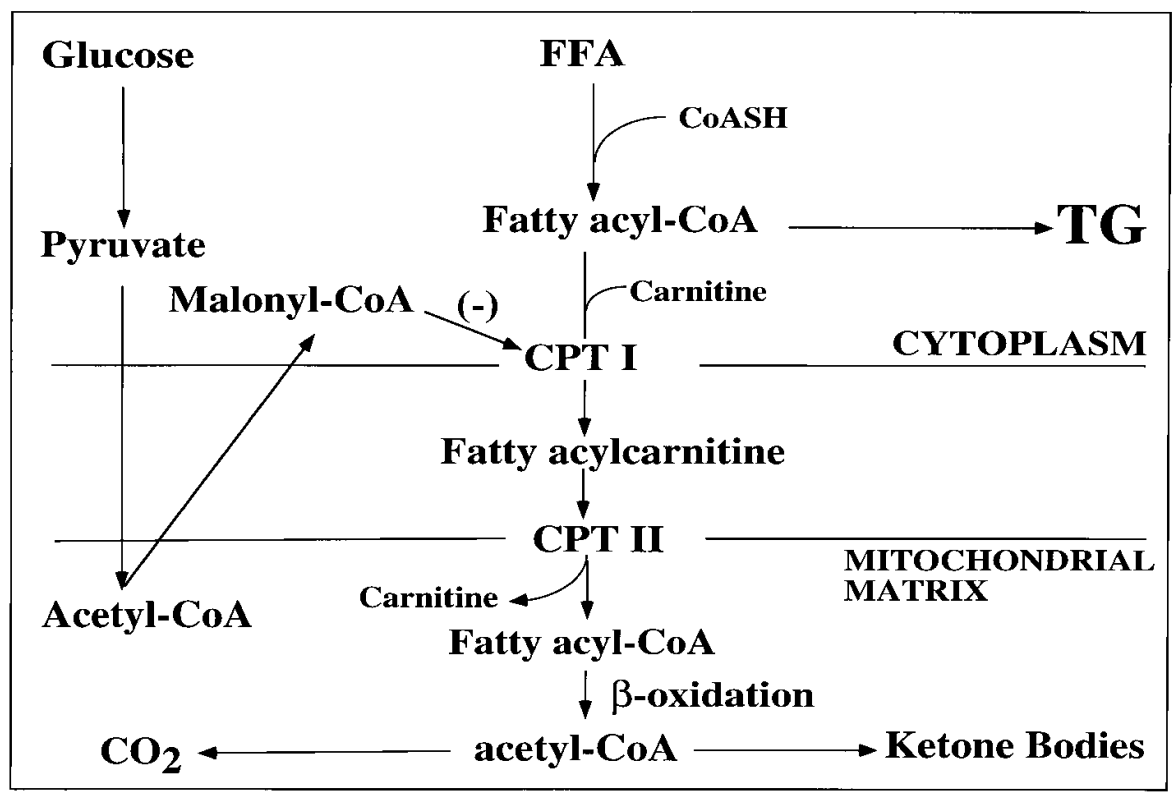

Figure 1. Schematic diagram showing the pathway of fatty acid oxidation in relation to glucose metabolism. ner mitochondrial membrane (11), these data suggested that limitations in the rate of translocation of LCFA across the mitochondrial membrane limited LCFA oxidation (14).

The notion that glucose and/or insulin might directly regulate fatty acid oxidation by determining the rate of LCFA uptake by the mitochondria has not been examined in human volunteers. In this study, we have tested the effect of the acute elevation of glucose availability and oxidation on fatty acid oxidation. We first directly assessed the effect of CPT I inhibition on LCFA and MCFA oxidation in an animal model. CPT I was inhibited using the pharmacologic inhibitor Etomoxir. The purpose of these studies was to determine if an agent known to inhibit CPT I would cause a selective reduction in LCFA, but not MCFA oxidation. We then used simultaneous infusions of $\left[1-{ }^{13} \mathrm{C}\right]$ oleate and $\left[1-{ }^{14} \mathrm{C}\right]$ octanoate to quantify LCFA and MCFA oxidation in human volunteers under basal conditions and during a hyperinsulinemic-hyperglycemic clamp. Plasma FFA availability was kept constant by the infusion of lipids and heparin. Muscle biopsies were obtained before and after the clamp to determine long-chain fatty acylcarnitine concentrations. A drop in long-chain acylcarnitine concentration during the clamp would further support the notion that glucose and/or insulin regulate fat oxidation by controlling the rate of long-chain fatty acyl-CoA entry into the mitochondrial matrix.

\section{Methods}

\section{Animal experiments}

Animals. Male Sprague-Dawley rats $(n=8,400-500$ grams) were purchased from Charles River (Portage, MI). All rats received water and food ad libitum and were maintained on a 12-h light/12-h dark cycle. All experiments were performed with the rats fasted for $12 \mathrm{~h}$. Rats received $1.0 \mathrm{ml} / \mathrm{kg}$ sodium pentobarbital for general anesthesia which was maintained throughout the study. Catheterization of the internal carotid artery and internal jugular vein on opposite sides of the neck was performed using 50-gauge polypropylene and 90-gauge silastic tubing, respectively. During the studies the rats were intermittently connected to artificial respiration (rodent ventilator, model 638; Harvard Apparatus, Inc., South Natick, MA) with tidal volume and rate set by normograms for the rat's weight.
Experimental design. Infusions of $\left[1-{ }^{14} \mathrm{C}\right]$ palmitate (constant infusion $=0.08 \mathrm{nCi} \cdot$ gram $\left.^{-1} \cdot \mathrm{min}^{-1}\right)($ DuPont-New England Nuclear Products, Inc., Boston, MA) dissolved in $0.9 \% \mathrm{NaCl}$, and $\left[1-{ }^{13} \mathrm{C}\right]$ octanoate (prime $=1.0 \mathrm{mg}$, constant infusion $=0.1 \mathrm{mg} / \mathrm{min}$ ) (Isotec Isotopes, Inc., Toronto, Canada) bound to $3 \%$ human albumin were started and maintained for $2 \mathrm{~h}$ using calibrated syringe pumps (basal period). After the basal period, at $2 \mathrm{~h}$ after the start of tracer infusion, a single dose of Etomoxir (10 mg) (gift from Byk Gulden, Konstanz, Germany) was given. Nicotinic acid boluses $(25 \mathrm{mg} / \mathrm{kg})$ were also given at 2,3, and $4 \mathrm{~h}$ after the start of tracer infusion to maintain plasma free fatty acid concentration to levels similar to the basal period.

Blood-breath sampling. Blood and breath samples were collected before tracer infusion and at 90, 100, 110, 120, 150, 180, 210, 240, 270, 300,330 , and $360 \mathrm{~min}$ after the start of tracer infusion. Expired air was collected by intubation via tracheotomy and 14-gauge Jelco catheter secured in place with 2-0 silk suture material for estimation of oxygen consumption $\left(\dot{\mathrm{VO}}_{2}\right)$ and carbon dioxide production $\left(\dot{\mathrm{VCO}}_{2}\right)$. Breath samples were analyzed for determination of ${ }^{13} \mathrm{CO}_{2}$ enrichment and ${ }^{14} \mathrm{CO}_{2}$ specific activity.

\section{Human experiments}

Volunteers. Seven volunteers, six males and one female (age $29 \pm 4 \mathrm{yr}$, weight $73 \pm 6 \mathrm{~kg}$, height $172 \pm 3 \mathrm{~cm}$ ), participated in one or more protocols of this study. The number of volunteers in each protocol is specified below. All volunteers were healthy, as indicated by comprehensive history, physical examination, and standard blood and urine tests. The studies were approved by the Institutional Review Board and the General Clinical Research Center of the University of Texas Medical Branch at Galveston.

Experimental design. All experiments were performed in the morning, after the volunteers had fasted overnight (i.e., $12 \mathrm{~h}$ ). Teflon catheters were placed percutaneously into an antecubital vein for isotope infusion and into a contralateral dorsal hand vein, which was heated, for sampling of arterialized venous blood. The catheters were kept patent by controlled infusion of $0.9 \% \mathrm{NaCl}$. The volunteers rested for $30 \mathrm{~min}$ after catheter placement, at which time one of the following three experimental protocols was performed.

Protocol 1 ( $n=7$, Fig. $2 A$ ): The first protocol consisted of two periods. In the first period (basal), the oxidation rates of oleate and octanoate tracers were quantified in the postabsorptive state. In the second period (i.e., hyperinsulinemic-hyperglycemic clamp [clamp]), the effect of accelerated carbohydrate flux on oleate and octanoate oxidation was determined. 


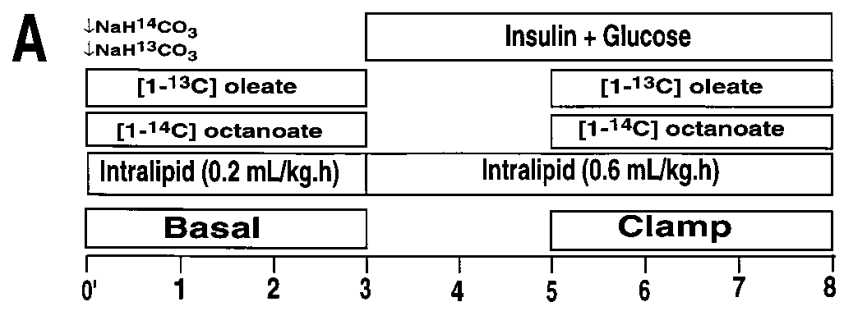

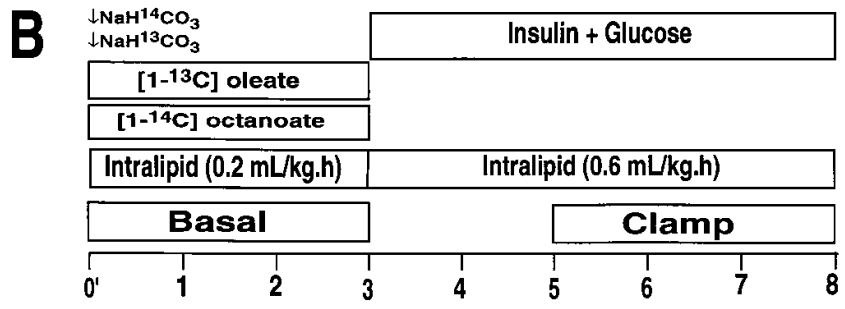

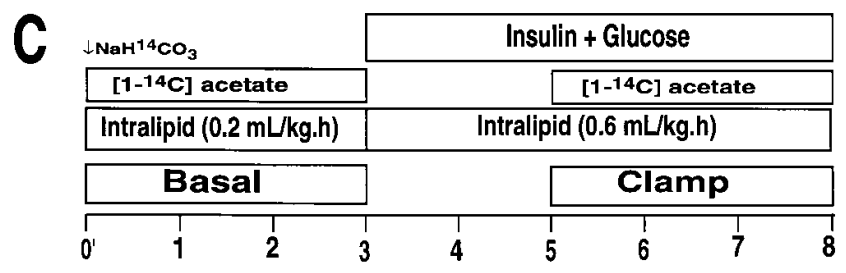

Figure 2. Schematic representation of study design. $A$, Protocol $1 ; B$, protocol 2; $C$, protocol 3.

Basal period (basal): Infusions of $\left[1-{ }^{14} \mathrm{C}\right]$ octanoate (prime $=4.0$ $\mathrm{nCi} / \mathrm{kg}$, constant infusion $=0.4 \mathrm{nCi} \cdot \mathrm{kg}^{-1} \cdot \min ^{-1}$ ) dissolved in $0.9 \%$ $\mathrm{NaCl}$, and $\left[1-{ }^{13} \mathrm{C}\right]$ oleate $\left(0.075 \mu \mathrm{mol} \cdot \mathrm{kg}^{-1} \cdot \mathrm{min}^{-1}\right)(99 \%$ enriched, MSD Isotopes, Montreal, Canada) bound to $5 \%$ human albumin were started and maintained for $3 \mathrm{~h}$ using calibrated syringe pumps. At the same time, a lipid emulsion (Intralipid ${ }^{\circledR}[20 \%]$, Kabi, Clayton, NC) $\left(0.2 \mathrm{ml} \cdot \mathrm{kg}^{-1} \cdot \mathrm{h}^{-1}\right)$, containing linoleic $(50 \%)$, oleic $(26 \%)$, palmitic $(10 \%)$, linolenic $(9 \%)$, and stearic $(3.5 \%)$ acids, was infused together with heparin (Elkins Sinn, Cherry Hill, NJ) (bolus of $3.0 \mathrm{U} /$ $\mathrm{kg}$; continuous infusion of $3.0 \mathrm{U} \cdot \mathrm{kg}^{-1} \cdot \mathrm{h}^{-1}$ ). Priming doses of $\mathrm{NaH}^{13} \mathrm{CO}_{3}(3.0 \mu \mathrm{mol} / \mathrm{kg})$ and $\mathrm{NaH}^{14} \mathrm{CO}_{3}(25 \mathrm{nCi} / \mathrm{kg})$ were given at the beginning of the studies, diluted in $0.01 \mathrm{~N} \mathrm{NaOH}$. The basal period lasted for $3 \mathrm{~h}$ at which time a muscle biopsy was performed. The biopsy sample $(\sim 100 \mathrm{mg})$ was obtained $15-20 \mathrm{~cm}$ above the patella from the vastus lateralis (lateral part). The muscle sample was immediately frozen and stored until further processing.

Hyperinsulinemic-hyperglycemic clamp (clamp): After the basal period, a primed $(1.5 \mathrm{U})$, continuous $\left(120 \mathrm{mU} \cdot \mathrm{m}^{-2} \cdot \mathrm{min}^{-1}\right)$ infusion of regular human insulin (Humulin R; Eli Lilly \& Co., Indianapolis, IN) was started and maintained for $5 \mathrm{~h}$. Plasma glucose concentration was clamped to $140 \mathrm{mg} / \mathrm{dl}$ for the duration of the clamp, using a variable infusion of $20 \%$ dextrose. A variable infusion of potassium chloride was also given to maintain plasma potassium levels. During the last $3 \mathrm{~h}$ of the clamp, $\left[1-{ }^{14} \mathrm{C}\right]$ octanoate and $\left[1-{ }^{13} \mathrm{C}\right]$ oleate were infused at the same rates used during the basal period. Lipids $\left(0.6 \mathrm{ml} \cdot \mathrm{kg}^{-1}\right.$. $\mathrm{h}^{-1}$ ) and heparin (bolus of $7.0 \mathrm{U} / \mathrm{kg}$; continuous infusion of $7.0 \mathrm{U}$. $\mathrm{kg}^{-1} \cdot \mathrm{h}^{-1}$ ) were infused throughout the clamp. A second muscle biopsy was obtained at the end of the study.

Protocol $2(n=7$, Fig. 2 B): The oxidation of glucose infused during the clamp to achieve the desired plasma glucose concentration is expected to interfere with isotopic estimations of fatty acid oxidation during the clamp, since it is established that glucose infusion may increase the carbon enrichment of expired $\mathrm{CO}_{2}(15)$. This is because cornstarch-derived glucose, which is commonly used in intravenous solutions in North America, has relatively high ${ }^{13} \mathrm{C}$ content (16). Thus, the purpose of protocol 2 was to quantify the effect of the oxidation of the infused glucose during the clamp, on breath ${ }^{13} \mathrm{C}$ enrichment. Similar to protocol 1, protocol 2 consisted of two periods: basal and clamp.

Basal period: Infusions of $\left[1-{ }^{14} \mathrm{C}\right]$ octanoate, $\left[1-{ }^{13} \mathrm{C}\right]$ oleate, lipid, and heparin, at the same infusion rates used in the basal period of protocol 1, were started and maintained for $3 \mathrm{~h}$. Priming doses of $\mathrm{NaH}^{13} \mathrm{CO}_{3}$ and $\mathrm{NaH}^{14} \mathrm{CO}_{3}$, as in protocol 1, were also given at the beginning of the studies. The basal period lasted for $3 \mathrm{~h}$.

Hyperinsulinemic-hyperglycemic clamp: After the basal period, a hyperinsulinemic-hyperglycemic clamp was started and maintained for $5 \mathrm{~h}$. Insulin, glucose, and lipids were infused at the same rates used during the clamp of protocol 1. For protocol 2, neither oleate nor octanoate tracers were infused during the clamp.

Protocol $3(n=3$, Fig. $2 C$ ): The purpose of protocol 3 was to determine the acetate-carbon recovery rate in the basal state and during the clamp. As we have suggested recently (17), the use of the acetate correction factor is preferable to the currently used bicarbonate correction factor for use in isotopic estimations of fatty acid oxidation. This is because, unlike the bicarbonate correction factor, the acetate correction factor fully accounts for label fixation, occurring at any point between the entrance of labeled acetyl-CoA into the tricarboxylic acid cycle and the appearance of labeled $\mathrm{CO}_{2}$ in breath $(17,18)$. Thus, on separate occasions, protocol 1 was repeated, without infusion of octanoate and oleate tracers. Instead, $\left[1-{ }^{14} \mathrm{C}\right]$ acetate (prime $=$ $5.0 \mathrm{nCi} / \mathrm{kg}$, constant infusion $=0.2 \mathrm{nCi} \cdot \mathrm{kg}^{-1} \cdot \mathrm{min}^{-1}$ ) was infused and the bicarbonate pool was primed using $\mathrm{NaH}^{14} \mathrm{CO}_{3}(10 \mathrm{nCi} / \mathrm{kg})$.

Blood-breath sampling. During both the basal and the clamp infusion periods, blood samples were collected before tracer infusion and at 30,60, 90,120,150,160,170, and $180 \mathrm{~min}$ after the start of tracer infusion. The blood was used for the determination of plasma oleate carbon enrichment and plasma FFA, triacylglycerol (TG), lactate, insulin, and glucose concentrations. Blood was not drawn during protocol 3. In all protocols, breath samples for determination of ${ }^{13} \mathrm{CO}_{2}$ enrichment and ${ }^{14} \mathrm{CO}_{2}$ specific activity were collected before tracer infusion and every $15 \mathrm{~min}$ throughout the tracer infusions. Indirect calorimetry was performed during the last $30 \mathrm{~min}$ of isotope infusion for estimation of $\dot{\mathrm{V}} \mathrm{O}_{2}$ and $\dot{\mathrm{V}} \mathrm{CO}_{2}$.

Assays. Expired air for measurement of ${ }^{14} \mathrm{CO}_{2}$ specific activity was collected in 3-liter anesthesia bags and $\mathrm{CO}_{2}$ was trapped by bubbling through a 1:4:9 solution of phenolphthalein $(0.1 \%$ solution; Fisher Scientific, Fair Lawn, NJ), benzethonium hydroxide (1.0 M solution; Sigma Chemical Co., St. Louis, MO), and absolute ethanol to trap exactly $1 \mathrm{mmol}$ of $\mathrm{CO}_{2}$. Scintillation fluid $(10 \mathrm{ml}$ of toluene, 0.04\% 2,5-diphenyl-oxazole; Sigma Chemical Co.) was added immediately in the vial and ${ }^{14} \mathrm{CO}_{2}$ specific activity (disintegrations $\cdot \mathrm{min}^{-1}$. $\mathrm{mmol}^{-1}[\mathrm{dpm} / \mathrm{mmol}]$ ) was determined using a liquid scintillation counter. $10 \mathrm{ml}$ of expired air was injected into evacuated tubes for determination of ${ }^{13} \mathrm{CO}_{2}$ to ${ }^{12} \mathrm{CO}_{2}$ ratio. Briefly, $\mathrm{CO}_{2}$ was isolated from the breath samples before analysis by isotope ratio mass spectrometry by passage through a water trap followed by condensation in a liquid nitrogen trap to allow other gases to be evacuated. The ${ }^{13} \mathrm{CO}_{2}$ to ${ }^{12} \mathrm{CO}_{2}$ ratio was then determined with a triple-collector isotope ratio mass spectrometer (SIRA VG Isotech, Cheshire, United Kingdom). The ratio is reported in units of tracer/tracee ratio (TTR) which is defined as: TTR $=\left({ }^{13} \mathrm{C} /{ }^{12} \mathrm{C}\right) \mathrm{sa}-\left({ }^{13} \mathrm{C} /{ }^{12} \mathrm{C}\right)$ ref $-\left[\left({ }^{13} \mathrm{C} /{ }^{12} \mathrm{C}\right) \mathrm{bk}-\left({ }^{13} \mathrm{C} /\right.\right.$ ${ }^{12} \mathrm{C}$ )ref]; where sa $=$ sample, ref $=$ reference gas, and $\mathrm{bk}=$ baseline sample.

Blood samples $(6 \mathrm{ml})$ were collected into prechilled tubes containing $120 \mu \mathrm{l}$ of $0.2 \mathrm{M}$ ethylene glycol-bis (b-aminoethyl ether)$N, N, N^{\prime}, N^{\prime}$-tetraacetic acid and plasma was immediately separated by centrifugation and frozen until further processing. This procedure has been shown to prevent in vitro lipolysis of TG in plasma from volunteers who were given heparin (19). Plasma oleate enrichment was determined by following previously described procedures (20). Briefly, FFA were extracted from plasma, isolated by thin layer chromatogra- 
phy, and converted to their methyl esters. The isotopic enrichment of oleate was determined by gas chromatography-mass spectrometry (model 5992; Hewlett-Packard Co., Palo Alto, CA), by selectively monitoring ions mass-to-charge of 296 and 297.

Plasma glucose concentration was measured on a glucose analyzer (2300 STAT; Yellow Springs Instrument Co., Yellow Springs, $\mathrm{OH}$ ) by use of the glucose oxidase method. Plasma lactate concentration was measured enzymatically on a lactate analyzer (2300 STAT; Yellow Springs Instrument Co.). Plasma TG concentration was measured enzymatically (RA-500; Technicon Instruments Corp., Tarrytown, NY). Plasma insulin concentration was determined using a radio-immunoassay method (INCSTAR, Stillwater, MN). Plasma oleate and total FFA concentrations were determined by gas chromatography (model 5890; Hewlett-Packard Co.), using heptadecanoic acid as internal standard. Plasma $\beta-\mathrm{OH}$-butyrate concentrations was determined enzymatically (Sigma Diagnostics).

The frozen muscle samples were weighed and powdered in a mortar and pestle cooled to the temperature of liquid $\mathrm{N}_{2}$. A portion of the powdered tissue was used to determine the dry-to-wet ratio of the samples. The CoA esters were extracted as described previously (21). The $6 \%$ perchloric acid extract was maintained at a $\mathrm{pH}$ of $2-3$. The CoA esters were measured using a modified HPLC procedure described by King et al. (22). Separation was performed on a Beckman System Gold with a ultraviolet detector 167. Each sample $(100 \mu \mathrm{l}$ each) was run through a precolumn cartridge (C18 size $3 \mathrm{~cm}, 7 \mu \mathrm{m})$ and a Microsorb short-one column (type C18, particle size $3 \mu \mathrm{m}$, size $4.6 \times 100 \mathrm{~mm}$ ). Absorbance was set at $254 \mathrm{~nm}$ and flow rate at $1 \mathrm{ml} /$ min. A gradient was initiated using two buffers: buffer A consisted of $0.2 \mathrm{M} \mathrm{NaH}_{2} \mathrm{PO}_{4}(\mathrm{pH} 5.0)$ and buffer $\mathrm{B}$ was a mixture of $0.25 \mathrm{M}$ $\mathrm{NaH}_{2} \mathrm{PO}_{4}$ and acetonitrile ( $\left.\mathrm{pH} 5.0\right)$ in a ratio of 80:20 (vol/vol). Buffers were filtered using filter pure, Nylon-66 filter membrane (Pierce, Rockford, IL). Initial conditions ( $97 \%$ A, 3\% B) were maintained for $2.5 \mathrm{~min}$ and were changed thereafter to $18 \% \mathrm{~B}$ over $5 \mathrm{~min}$ using Beckman's curve 3 . At 15 min the gradient was changed linearly to $37 \%$ B over 3 min and subsequently to $90 \%$ B over $17 \%$ min. At 42 min, the composition was returned linearly back to $3 \%$ B over 0.5 $\mathrm{min}$, and at 50 min column equilibration was complete. Peaks were integrated by Beckman System Gold software package.

Long-chain acyl-CoA and long-chain acylcarnitine were extracted and measured in the acid-insoluble perchloric acid fraction as described previously (21). Extracted long-chain acyl-CoA was hydrolyzed and free CoA was measured fluorometrically (21). Extracted long-chain acylcarnitine was also hydrolyzed and free carnitine was measured radiometrically (12).

Calculations. The rate of appearance $\left(R_{\mathrm{a}}\right)$ of oleate in plasma, which under steady state conditions is equal to the rate of disappearance $\left(R_{\mathrm{d}}\right)$, was calculated as: $R_{\mathrm{a}}=\mathrm{F} / \mathrm{Eo}$; where $\mathrm{F}=$ oleate tracer infusion rate, and Eo = oleate carbon enrichment in plasma. FFA $R_{\mathrm{a}}$ was derived by dividing the $R_{\mathrm{a}}$ oleate by the fractional contribution of oleate to the total FFA concentration, as determined by gas chromatography.

The rates of ${ }^{13} \mathrm{CO}_{2}$ and ${ }^{14} \mathrm{CO}_{2}$ excretion, resulting from the oxidation of $\left[1-{ }^{13} \mathrm{C}\right]$ oleate and $\left[1-{ }^{14} \mathrm{C}\right]$ octanoate, respectively, were calculated as: labeled $\mathrm{CO}_{2}$ excretion $=\dot{\mathrm{V} C O} 2 \times \mathrm{ECO}_{2}$; where $\mathrm{ECO}_{2}=$ breath $\mathrm{CO}_{2}$ carbon enrichment.

Carbohydrate and total fatty acid oxidation rates were calculated from the indirect calorimetry data using stoichiometric equations (23). $\dot{\mathrm{V}} \mathrm{O}_{2}$ and $\dot{\mathrm{VCO}}_{2}$ values were the average over 20 min of steady state measurements. Fatty acid oxidation was determined by converting the rate of TG oxidation ( $\mathrm{grams} \cdot \mathrm{kg}^{-1} \cdot \mathrm{min}^{-1}$ ) to its molar equivalent, assuming the average molecular weight of TG to be 860 grams/ mol (23), and multiplying the molar rate of TG oxidation by three because each molecule contains $3 \mathrm{~mol}$ of fatty acids.

The absolute rates of $\left[1-{ }^{13} \mathrm{C}\right]$ oleate and $\left[1-{ }^{14} \mathrm{C}\right]$ octanoate oxidation $\left(\mu \mathrm{mol} \cdot \mathrm{kg}^{-1} \cdot \mathrm{min}^{-1}\right)$ were calculated as: substrate oxidation $=$ $\left.\left(\mathrm{ECO}_{2} \times \dot{\mathrm{V} C O}\right)_{2}\right) /(\mathrm{Eo}) \times$ ar; where ar is the acetate correction factor determined in protocol 3 . The specific activity of the octanoate infusion mixture was used as the octanoate enrichment in plasma since octanoate is not produced endogenously. Plasma fatty acid oxidation was determined by dividing the $R_{\mathrm{a}}$ oleate by the fractional contribution of oleate to the total FFA concentration, as determined by gas chromatography.

For the determination of breath carbon enrichment $\left({ }^{13} \mathrm{C}\right.$ to ${ }^{12} \mathrm{C}$ and ${ }^{14} \mathrm{C}$ to ${ }^{12} \mathrm{C}$ ratios) during the clamp of protocol 1 , the values for breath carbon enrichment obtained during the clamp of protocol 2 were used as the background value (see experimental protocol 2).

Statistical analysis. Differences between plasma oleate enrichment, plasma TG, glucose, insulin, and lactate concentrations, longchain acylcarnitine concentrations and the calculated rates of fatty acid kinetics and oxidation in the basal and clamp periods of protocol 1 were identified using a paired $t$ test. Statistical significance was considered present if $P<0.05$. Values are presented as means \pm SE.

\section{Results}

\section{Animal experiments}

In preliminary experiments it was noted that administration of Etomoxir results in significant increase in plasma FFA concentration. By using nicotinic acid, we were able to maintain a stable plasma FFA concentration throughout the experiment (data not shown).

Addition of Etomoxir, a CPT I specific inhibitor, resulted in a significant decrease in palmitate-derived ${ }^{14} \mathrm{CO}_{2}$ specific activity and palmitate oxidation (Fig. 3). In contrast, octanoatederived ${ }^{13} \mathrm{CO}_{2}$ enrichment and octanoate oxidation did not change after administration of Etomoxir. These results confirm the notion that an inhibition of CPT I activity is readily reflected in changes in LCFA but not MCFA oxidation.

\section{Human experiments}

Protocol 1. Plasma FFA and TG concentrations were similar during basal and clamp, whereas plasma $\beta-\mathrm{OH}$-butyrate concentrations decreased during the clamp to levels that were generally undetectable (Table I). Plasma insulin, glucose, and lactate concentrations were significantly higher during the clamp compared with the basal period (Table I).

Plasma oleate enrichments were similar during basal and clamp $\left(0.043 \pm 0.01\right.$ and $0.042 \pm 0.01$, respectively). $R_{\mathrm{a}}$ oleate re-

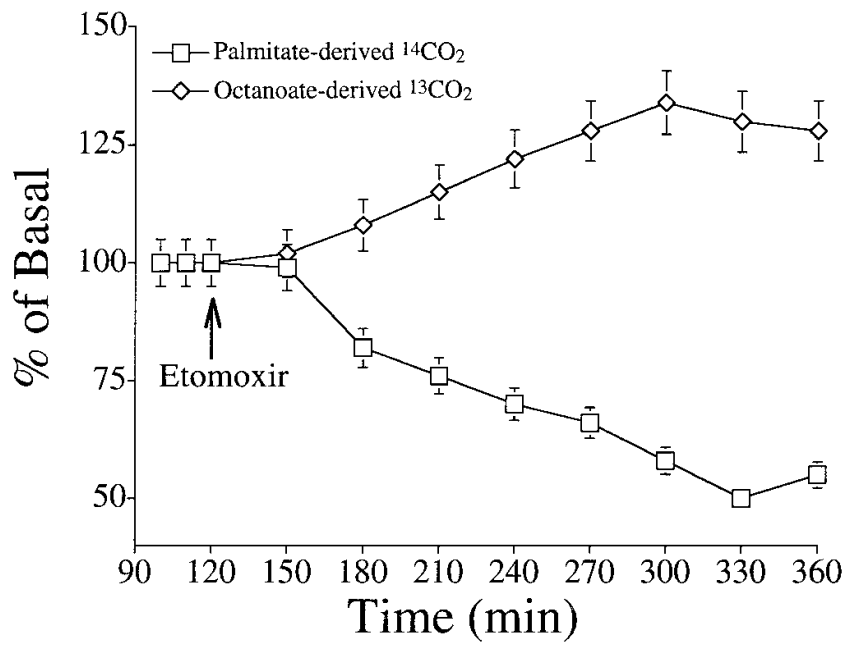

Figure 3. Effect of Etomoxir on rat breath palmitate-derived ${ }^{14} \mathrm{CO}_{2}$ specific activity and octanoate-derived ${ }^{13} \mathrm{CO}_{2}$ enrichment. Data are presented as percentage of the values obtained during the control period. Means \pm SE for eight animals. 
Table I. Substrate and Hormone Concentrations at the Basal State and During Hyperinsulinemia-Hyperglycemia (Protocol 1)

\begin{tabular}{lcc}
\hline & Basal & Clamp \\
\hline TG (mmol/liter) & $1.7 \pm 0.5$ & $2.0 \pm 0.4$ \\
FFA (mmol/liter) & $0.46 \pm 0.06$ & $0.39 \pm 0.07$ \\
Glucose (mmol/liter) & $4.6 \pm 0.2$ & $7.6 \pm 0.1^{*}$ \\
Lactate (mmol/liter) & $0.8 \pm 0.1$ & $1.7 \pm 0.1^{*}$ \\
Insulin (pmol/liter) & $32 \pm 4$ & $1526 \pm 139^{*}$ \\
$\beta$-OH-butyrate (mmol/liter) & $0.27 \pm 0.12$ & $0.06 \pm 0.06^{*}$ \\
& &
\end{tabular}

Values are means \pm SE for seven volunteers, $* P<0.05$ vs. basal.

mained unchanged during the clamp (Table II). Isotopically determined plasma oleate oxidation decreased by $43 \%$ during the clamp (Table II, Fig. 4). Unlike oleate, octanoate oxidation did not change significantly during the clamp (Table II, Fig. 4).

Indirect calorimetry data indicated a significant increase in total carbohydrate oxidation from $5.3 \pm 1.5$ during basal to $26.1 \pm 3.3 \mu \mathrm{mol} \cdot \mathrm{kg}^{-1} \cdot \mathrm{min}^{-1}$ during the clamp. Correspondingly, plasma fatty acid oxidation (tracer methodology) and total fatty acid oxidation (indirect calorimetry) significantly decreased during the clamp (Table II).

The long-chain acylcarnitine concentration obtained from the vastus lateralis muscle at the end of the clamp was lower $(P<0.05)$ than the concentration at the end of the basal period (Fig. 5). This is consistent with the lower oleate oxidation rates during the clamp. Long-chain acyl-CoA, which is present in both the cytoplasm and the inner mitochondrial matrix, did not differ between the basal and the clamp conditions $(40.4 \pm 22.4$ and $33.6 \pm 12.3 \mathrm{nmol} / \mathrm{gram}$ dry weight, under basal and clamp conditions, respectively). The amount of free, nonesterified CoA also did not differ $(61.4 \pm 16.6$ and $59.5 \pm 12.2$ $\mathrm{nmol} / \mathrm{gram}$ dry weight, under basal and clamp conditions, respectively), nor did the levels of acetyl-CoA (1.1 \pm 0.6 and $1.1 \pm 0.5 \mathrm{nmol} /$ gram dry weight). Attempts were also made to measure malonyl-CoA levels; however accurate measurements were not possible. This was due in part to the small amount of biopsy tissue available as well as in limitations in the sensitivity of the HPLC assay.

Table II. Plasma and Total Fatty Acid Kinetics and Oxidation at the Basal State and During Hyperinsulinemia-Hyperglycemia (Protocol 1)

\begin{tabular}{lcc}
\hline & Basal & Clamp \\
\hline Oleate $R_{\mathrm{a}}$ & $1.8 \pm 0.3$ & $1.9 \pm 0.3$ \\
FFA $R_{\mathrm{a}}$ & $4.7 \pm 0.6$ & $6.3 \pm 1.4$ \\
Oleate oxidation & $0.7 \pm 0.1$ & $0.4 \pm 0.1^{*}$ \\
Octanoate oxidation & $4.0 \mathrm{e}^{-06} \pm 3.2 \mathrm{e}^{-07}$ & $4.3 \mathrm{e}^{-06} \pm 4.0 \mathrm{e}^{-07}$ \\
Plasma fatty acid oxidation & $2.4 \pm 0.3$ & $1.4 \pm 0.1^{*}$ \\
Total fatty acid oxidation & $3.0 \pm 0.4$ & $0.4 \pm 0.3^{*}$ \\
& & \\
\hline
\end{tabular}

Values are means $\pm \mathrm{SE}$ for seven volunteers expressed in $\mu \mathrm{mol} \cdot \mathrm{kg}^{-1}$. $\min ^{-1}$. Indirect calorimetry significantly underestimated fatty acid oxidation during the clamp. This explains the fact that total fatty acid oxidation (indirect calorimetry) was lower than plasma fatty acid oxidation (tracer methodology). ${ }^{*} P<0.05$ vs. basal.

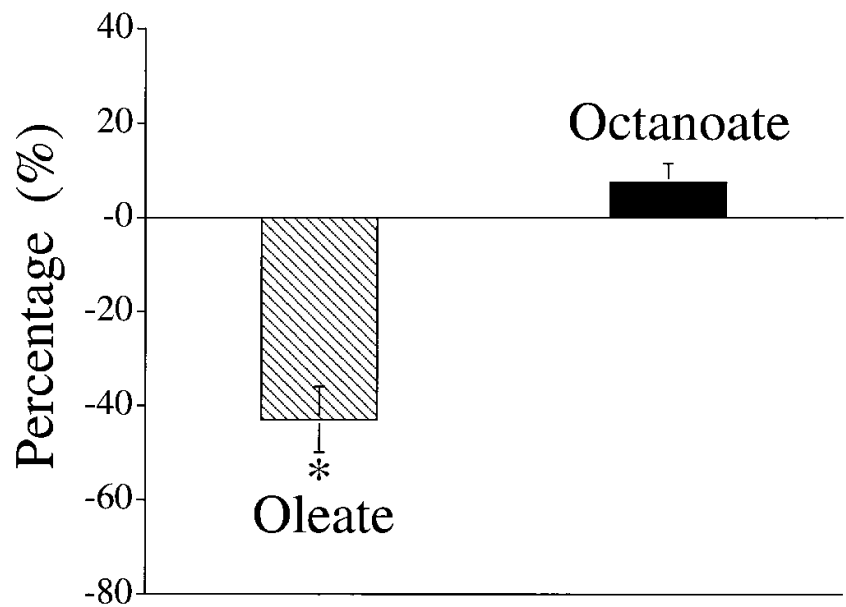

Figure 4. Percent difference in $\left[1-{ }^{13} \mathrm{C}\right]$ oleate and $\left[1-{ }^{14} \mathrm{C}\right]$ octanoate oxidation in the transition from the basal state to hyperinsulinemiahyperglycemia (protocol 1 ). Means \pm SE for seven volunteers, $* P<0.05$ from baseline.

Protocol 2. $5 \mathrm{~h}$ after stopping the $\left[1-{ }^{13} \mathrm{C}\right]$ oleate infusion (i.e., in the final minutes of the clamp), breath ${ }^{13} \mathrm{C}$ enrichment was still $\sim 45 \%$ of the plateau ${ }^{13} \mathrm{C}$ enrichment during the basal state; from $1.6 \mathrm{e}^{-04} \pm 1.0 \mathrm{e}^{-05}$ during basal decreased to $7.5 \mathrm{e}^{-05} \pm$ $4.9 \mathrm{e}^{-06}$ during the clamp $(P<0.05$ vs. the plateau enrichment during basal and versus the background enrichment before tracer infusion started). The persistent elevation of ${ }^{13} \mathrm{C}$ enrichment during the clamp, despite the fact that no labeled oleate was infused, reflected the effect of unlabeled glucose infusion. In contrast, in the final minutes of the clamp, breath ${ }^{14} \mathrm{CO}_{2}$ enrichment was only $\sim 10 \%$ of the plateau ${ }^{14} \mathrm{CO}_{2}$ enrichment during basal. The values for breath carbon enrichment obtained during the clamp of protocol 2 were used as the background values for the determination of breath carbon enrichment during the clamp of protocol 1 .

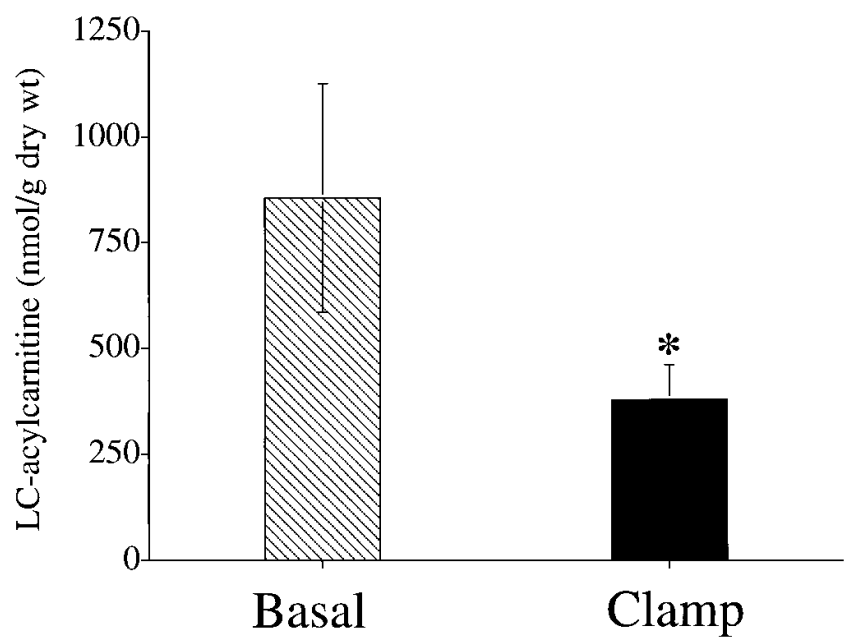

Figure 5. Long-chain acylcarnitine concentration in the basal state and after $5 \mathrm{~h}$ of hyperinsulinemia-hyperglycemia (protocol 1). The biopsy samples were taken from the vastus lateralis muscle. Means \pm SE for seven volunteers, $* P<0.05$ from basal. 
Protocol 3. Acetate-labeled carbon recovery in breath $\mathrm{CO}_{2}$ was $55.1 \pm 2.2 \%$ (basal) and $50.3 \pm 3.4 \%$ (clamp) of the infused label.

\section{Discussion}

In this study we have confirmed our recent finding (9) that glucose plus insulin decrease plasma LCFA oxidation even when fatty acid availability is maintained constant, via infusion of exogenous lipids (Table II, Fig. 4). This finding suggests that accelerated carbohydrate oxidation and/or insulin directly inhibits fatty acid oxidation by a mechanism other than inhibition of lipolysis. In contrast to the effect of glucose on LCFA oxidation, glucose plus insulin infusion had no effect on MCFA oxidation. Since the only known difference in the oxidation patterns of oleate and octanoate is the requirement of the former for CPT I in order to gain access into the mitochondria, the inhibition of oleate but not octanoate oxidation suggests that hyperinsulinemia-hyperglycemia regulates fatty acid oxidation by controlling the rate of LCFA uptake by the mitochondria. This notion is supported by the decrease in long-chain acylcarnitine levels in skeletal muscle during the clamp (Fig. 5), a response expected if CPT I was inhibited.

Unlike oleate, octanoate is not found in measurable quantities in human plasma. Thus, in the present studies octanoate was only available at trace amounts, whereas oleate concentration was significantly higher. To determine if the difference in the relative concentrations between oleate and octanoate could account for the observed differences in their oxidation rates during the clamp, in two of the studies of protocol 1 we infused unlabeled octanoate at a rate to match the $R_{\mathrm{a}}$ of endogenous oleate. Octanoate tracer oxidation increased from $3.8 \mathrm{e}^{-06} \mu \mathrm{mol} \cdot \mathrm{kg}^{-1} \cdot \mathrm{min}^{-1}$ in the basal state to $3.3 \mathrm{e}^{-06} \mu \mathrm{mol}$. $\mathrm{kg}^{-1} \cdot \min ^{-1}$ during the clamp $(n=2)$, suggesting that the differences observed between octanoate and oleate tracer oxidation were not due to differences in their relative concentrations. Thus, means of all seven studies are presented throughout the manuscript.

The comparison of octanoate oxidation with oleate oxidation requires the assumption that the only difference between the fate of the two tracers is the need for oleate to use the CPT I for transport across the mitochondrial membrane. McGarry et al. (24) tested this assumption by comparing relative rates of oxidation of $(-)$-octanoylcarnitine, octanoate, and oleate in perfused rat livers. (-)-Octanoylcarnitine behaves as an LCFA, with regard to transport across the mitochondria, and as an MCFA with regard to $\beta$-oxidation. It was found that the relative rate of ketone body production from (-)-octanoylcarnitine followed that observed for oleate rather than octanoate in livers from fed and fasted rats, suggesting that regulation of fat oxidation is exerted in the CPT I step. These findings support the assumption that the only difference in the oxidation of oleate and octanoate is the need for oleate to use the CPT I for transport across the mitochondrial membrane.

The finding that overall tissue levels of long-chain fatty acyl-CoA did not change during the clamp suggests that fatty acid availability to acyl-CoA synthetase was not compromised during the clamp. Thus, the effect of accelerated glucose metabolism on fatty acid oxidation was not likely on fatty acid uptake but it was exerted either at the acyl-CoA synthetase or the CPT I steps. The former possibility is unlikely, since inhibition of oleate oxidation by malonyl-CoA in rat livers resulted in a marked increase in the incorporation of oleate into TG (10). This would not be possible if there was a limitation in the fatty acyl-CoA synthetase step. Thus, from the above, we conclude that inhibition of LCFA, but not MCFA oxidation during the clamp was exerted at the CPT I step. This is supported by the observed significant decrease in long-chain acylcarnitine during the clamp (Fig. 5).

The mechanism by which CPT I activity was decreased during the clamp is not proven in this study. One possibility is a direct insulin-induced increase in malonyl-CoA levels. In tissues such as liver and adipose tissue, insulin stimulates acetyl-CoA carboxylase, the enzyme which produces malonyl-CoA (25). Increased glucose metabolism could also increase malonylCoA production by increasing the supply of acetyl-CoA. Recent studies suggest that when glucose oxidation increases, acetyl groups can be translocated from acetyl-CoA within the mitochondrial matrix to acetyl-CoA into the cytoplasm (for review see reference 26). In rat heart, stimulation of glucose oxidation increased malonyl-CoA production, which was correlated with a decrease in LCFA oxidation (27). In that study (27), as in this study, the increase in glucose oxidation after the clamp was coupled with a decrease in tissue long-chain acylcarnitine concentration, suggesting a decrease in fatty acid uptake by the mitochondria in the muscle. Further, the decrease in plasma $\beta-\mathrm{OH}$-butyrate concentration during the clamp (Table I) suggests that accelerated glucose metabolism, or insulin per se, also affected hepatic fatty acid oxidation.

Another possibility that needs to be considered is that stimulation of glucose oxidation may result in accumulation of cytosolic acetylcarnitine, thereby decreasing the availability of free carnitine for CPT I activity. A decrease in cytosolic carnitine levels has the potential to limit CPT I activity, and, thus fatty acid oxidation, since skeletal muscle predominately expresses an $82-\mathrm{kD}$ isoform of CPT I which has a low affinity for L-carnitine (12). However, no study has yet convincingly demonstrated that an increased esterification of cytosolic L-carnitine can limit CPT I activity.

There was a large discrepancy between fatty acid oxidation calculated using indirect calorimetry and tracer methodology, during the clamp in protocol 1 (Table II). Indirect calorimetry suggests that there is virtually no fatty acid oxidation during the clamp. However, tracer estimates of plasma fatty acid oxidation indicate that fatty acid oxidation, although lower than during basal, was nevertheless significant during the clamp. The validity of indirect calorimetry as a direct index of substrate oxidation has been questioned under conditions such as hyperinsulinemia-hyperglycemia where lipogenesis might occur (28). Tracer estimation of plasma fatty acid oxidation is probably the only means for accurate determination of fatty acid oxidation under these circumstances, providing that account is taken of factors which might affect labeled $\mathrm{CO}_{2}$ excretion (e.g., slow labeled $\mathrm{CO}_{2}$ decay, contribution of naturally enriched glucose [if ${ }^{13} \mathrm{C}$ fatty acid tracers are used], label fixation in the TCA cycle and the bicarbonate pool) $(17,18)$. Although tracer techniques reflect only plasma fatty acid oxidation, we have shown recently that, at rest, nearly all fatty acids used for oxidation come from the blood $(17,18)$.

In summary, these results suggest that in addition to the well documented regulation of FFA oxidation by insulin via its antilypolytic effect, glucose plus insulin directly regulate fatty acid oxidation by controlling the rate of LCFA entrance into the mitochondria. 


\section{Acknowledgments}

The authors gratefully appreciate the help of the nursing staff of the General Clinical Research Center in the performance of the experiments. We thank Dr. S. Klein and Dr. B. Williams for the performance of the biopsies, and Dr. Y. Zheng, Dr. Amalia Gastaldelli, Dr. John Kirk, Y.-X. Lin, Y. Sun, and G. Jones for excellent technical assistance.

This work was supported by National Institutes of Health grants DK-34817 and DK-46017, General Clinical Research Center grant 00073, and Shriners Hospital grant 15849.

\section{References}

1. Randle, P.J., P.B. Garland, C.N. Hales, and E.A. Newsholme. 1963. The glucose-fatty acid cycle: its role in insulin sensitivity and the metabolic disturbances of diabetes mellitus. Lancet. 1:785-789.

2. Bevilacqua, S., R. Bonadonna, G. Buzzigoli, C. Boni, D. Ciociaro, F. Maccari, M.-A. Giorico, and E. Ferrannini. 1987. Acute elevation of free fatty acid levels leads to hepatic insulin resistance in obese subjects. Metab. Clin. Exp. 36:502-506.

3. Boden, G., X. Chen, J. Ruiz, J.V. White, and L. Rossetti. 1994. Mechanisms of fatty acid-induced inhibition of glucose uptake. J. Clin. Invest. 93: 2438-2446.

4. Ferrannini, E., E.J. Barrett, S. Bevilacqua, and R.A. DeFronzo. 1983. Effect of fatty acids on glucose production and utilization in man. J. Clin. Invest. 72:1737-1747.

5. Saloranta, C., V. Koivisto, E. Widen, K. Falholt, R.A. DeFronzo, M. Harkonen, and L.C. Groop. 1993. Contribution of muscle and liver to glucosefatty acid cycle in humans. Am. J. Physiol. 264:E599-E605.

6. Wolfe, B.M., S. Klein, E.J. Peters, B.F. Schmidt, and R.R. Wolfe. 1988. Effect of elevated free fatty acids on glucose oxidation in normal humans. Metab. Clin. Exp. 37:323-329.

7. Yki-Yärvinen, H., I. Puhakainen, and V.A. Koivisto. 1991. Effect of free fatty acids on glucose uptake and nonoxidative glycolysis across human forearm tissues in the basal state and during insulin stimulation. J. Clin. Endocrinol. \& Metab. 72:1268-1277.

8. Wolfe, R.R., and E.J. Peters. 1987. Lipolytic response to glucose infusion in human subjects. Am. J. Physiol. 252:E218-E223.

9. Sidossis, L.S., and R.R. Wolfe. 1996. Glucose and insulin-induced inhibition of fatty acid oxidation: the glucose-fatty acid cycle reversed. Am. J. Physiol. 270:E733-E738.

10. McGarry, J.D., G.P. Mannaerts, and D.W. Foster. 1977. A possible role for malonyl-CoA in the regulation of hepatic acid oxidation and ketogenesis. $J$. Clin. Invest. 60:265-270.

11. Fritz, I.B. 1959. Action of carnitine on long chain fatty acid oxidation by liver. Am. J. Physiol. 197:297-304.

12. McGarry, J.D., S.E. Milles, C.S. Long, and D.W. Foster. 1983. Observations on the affinity for carnitine, and malonyl-CoA sensitivity, of carnitine palmitoyltransferase I in animal and human tissues. Biochem. J. 214:21-28.

13. McGarry, J.D., K.F. Woeltje, M. Kuwajima, and D.W. Foster. 1989. Regulation of ketogenesis and the renaissance of carnitine palmitoyltransferase. Diabetes Metab. Rev. 5:271-284.

14. McGarry, J.D., and D.W. Foster. 1971. The regulation of ketogenesis from octanoic acid. The role of the tricarboxylic acid cycle and fatty acid synthesis. J. Biol. Chem. 246:1149-1159.

15. Wolfe, R.R., J.H. Shaw, E.R. Nadel, and M.H. Wolfe. 1984. Effect of substrate intake and physiological state on background ${ }^{13} \mathrm{CO}_{2}$ enrichment. $J$. Appl. Physiol. 56:230-234.

16. Schoeller, D.A., P.D. Klein, J.B. Watkins, T. Heim, and J. Machean. 1980. ${ }^{13} \mathrm{C}$ abundances of nutrients and the effect of variations in ${ }^{13} \mathrm{C}$ isotope abundances of test meals formulated for ${ }^{13} \mathrm{CO}_{2}$ breath tests. Am. J. Clin. Nutr. 33:2375-2385.

17. Sidossis, L.S., A.R. Coggan, A. Gastaldelli, and R.R. Wolfe. 1995. A new correction factor for use in tracer estimations of plasma fatty acid oxidation. Am. J. Physiol. 269:E649-E656.

18. Sidossis, L.S., A.R. Coggan, A. Gastaldelli, and R.R. Wolfe. 1995. Pathway of free fatty acid oxidation in human subjects. Implications for tracer studies. J. Clin. Invest. 95:278-284.

19. Hargreaves, M., B. Kiens, and E.A. Richter. 1991. Effect of increased plasma free fatty acid concentrations on muscle metabolism in exercising men. J. Appl. Physiol. 70:194-201.

20. Wolfe, R.R. 1992. Radioactive and Stable Isotope Tracers in Biomedicine. Wiley-Liss, New York. 422 pp.

21. Idell-Wenger, J.A., L.W. Grotyohann, and J.R. Neely. 1978. Coenzyme A and carnitine distribution in normal and ischemic hearts. J. Biol. Chem. 253: 4310-4318.

22. King, M.T., P.D. Reiss, and N.W. Cornell. 1988. Determination of shortchain coenzyme A compounds by reversed-phase high-performance liquid chromatography. Methods Enzymol. 166:70-79.

23. Frayn, K.H. 1983. Calculation of substrate oxidation rates in vivo from gaseous exchange. J. Appl. Physiol. 55:628-634.

24. McGarry, J.D., and D.W. Foster. 1974. The metabolism of (-)-octanoylcarnitine in perfused livers from fed and fasted rats: evidence for a possible regulatory role of carnitine acyltransferase in the control of ketogenesis. J. Biol. Chem. 249:7984-7990.

25. Hardie, D.G. 1989. Regulation of fatty acid synthesis via phosphorylation of acetyl-CoA carboxylase. Prog. Lipid Res. 28:117-146.

26. Lopaschuk, G.D., D.D. Belke, J. Gamble, T. Itoi, and B. Schonekess 1994. Regulation of fatty acid oxidation in the mammalian heart in health and disease. Biochim. Biophys. Acta. 1213:263-276.

27. Saddik, M., J. Gamble, L.A. Witters, and G.D. Lopaschuk. 1993. Acetyl-CoA carboxylase regulation of fatty acid oxidation in the heart. J. Biol. Chem. 268:25836-25845.

28. Simonson, D.C., and R.A. DeFronzo. 1990. Indirect calorimetry: methodological and interpretative problems. Am. J. Physiol. 258:E399-E412. 